\title{
Real-Life Impact of Early Interferon $\beta$ Therapy in Relapsing Multiple Sclerosis
}

\author{
M. Trojano, MD, ${ }^{1}$ F. Pellegrini, MDeStat, ${ }^{2}$ D. Paolicelli, MD, ${ }^{1}$ A. Fuiani, MD, ${ }^{1}$ G.B. Zimatore, MD,${ }^{1}$ \\ C. Tortorella, MD, ${ }^{1}$ I.L. Simone, MD, ${ }^{1}$ F. Patti, MD, ${ }^{3}$ A. Ghezzi, MD, ${ }^{4}$ V. Zipoli, MD, ${ }^{5}$ P. Rossi, MD, ${ }^{6}$ \\ C. Pozzilli, MD, ${ }^{7}$ G. Salemi, MD, ${ }^{8}$ A. Lugaresi, MD, ${ }^{9}$ R. Bergamaschi, MD,${ }^{10}$ E. Millefiorini, MD,${ }^{11}$ \\ M. Clerico, MD, ${ }^{12}$ G. Lus, MD, ${ }^{13}$ M. Vianello, MD, ${ }^{14}$ C. Avolio, MD, ${ }^{15}$ P. Cavalla, MD, ${ }^{16}$ V. Lepore, MD, ${ }^{2}$ \\ P. Livrea, MD, ${ }^{1}$ G. Comi, MD, ${ }^{6}$ and M.P. Amato, $\mathrm{MD}^{5}$; for the Italian Multiple Sclerosis Database Network \\ (MSDN) Group
}

\begin{abstract}
Objective: Recent findings support greater efficacy of early vs. delayed interferon beta (IFN $\beta$ ) treatment in patients with a first clinical event suggestive of multiple sclerosis (MS). We aimed to evaluate the effectiveness of early IFN $\beta$ treatment in definite relapsing-remitting MS (RRMS) and to assess the optimal time to initiate IFN $\beta$ treatment with regard to the greatest benefits on disability progression.

Methods: A cohort of 2,570 IFN $\beta$-treated RRMS patients was prospectively followed for up to 7 years in 15 Italian MS Centers. A Cox proportional hazards regression model adjusted for propensity score (PS) quintiles was used to assess differences between groups of patients with early vs. delayed IFN $\beta$ treatment on risk of reaching a 1-point progression in the Expanded Disability Status Scale (EDSS) score, and the EDSS 4.0 and 6.0 milestones. A set of PS-adjusted Cox hazards regression models were calculated according to different times of treatment initiation (within 1 year up to within 5 years from disease onset). A sensitivity analysis was performed to assess the robustness of findings.

Results: The lowest hazard ratios (HRs) for the three PS quintiles-adjusted models were obtained by a cutoff of treatment initiation within 1 year from disease onset. Early treatment significantly reduced the risk of reaching a 1-point progression in EDSS score $(\mathrm{HR}=0.63 ; 95 \% \mathrm{CI}=0.48-0.85 ; p<0.002)$, and the EDSS 4.0 milestone $(\mathrm{HR}=0.56 ; 95 \% \mathrm{CI}=0.36-$ $0.90 ; p=0.015)$. Sensitivity analysis showed the bound of significance for unmeasured confounders.
\end{abstract}

Interpretation: Greater benefits on disability progression may be obtained by an early IFN $\beta$ treatment in RRMS.

Ann Neurol 2009;66:513-520

The role of interferon beta (IFN $\beta$ ) as a diseasemodifying drug for the treatment of relapsingremitting multiple sclerosis (RRMS) is now well estab- lished, and its efficacy has been demonstrated in randomized controlled trials (RCTs). ${ }^{1-3}$ The long-term effectiveness of IFN $\beta$ on delaying irreversible clinical
From the ${ }^{1}$ Department of Neurological and Psychiatric Sciences, University of Bari, Bari, Italy; ${ }^{2}$ Department of Clinical Pharmacology and Epidemiology, Consorzio Mario Negri Sud, Santa Maria Imbaro, Chieti, Italy; ${ }^{3}$ Department of Neurology, University of Catania, Catania, Italy; ${ }^{4}$ Multiple Sclerosis Centre, Hospital of Gallarate, Gallarate, Italy; ${ }^{5}$ Department of Neurology, University of Florence, Florence, Italy; ${ }^{6}$ Department of Neurology, Hospital of San Raffaele, Milan, Italy; ${ }^{7}$ Multiple Sclerosis Center, S. Andrea Hospital, University of Rome "La Sapienza," Rome, Italy; ${ }^{8}$ Department of Clinical Neurosciences, University of Palermo, Palermo, Italy; ${ }^{9}$ Department of Oncology and Neuroscience, University G. d'Annunzio, Chieti, Italy; ${ }^{10}$ Multiple Sclerosis Center, Neurological Institute C. Mondino, Pavia, Italy; ${ }^{11}$ Department of Neurological Sciences, University "La Sapienza," Rome, Italy; ${ }^{12}$ Department of Neurology, S. Luigi Gonzaga Hospital, University of Turin, Turin, Italy; ${ }^{13}$ Department of Neurological Sciences, 2nd University of Naples, Naples, Italy; ${ }^{14} \mathrm{O}$. U. Neurology, Ca' Foncello Hospital, Treviso, Italy; ${ }^{15}$ Department of Medical and Occupational Sciences, University of Foggia, Foggia, Italy; ${ }^{16}$ Department of Neuroscience, University of Turin, Turin, Italy

Address correspondence to Professor Maria Trojano, Department of Neurological and Psychiatric Sciences, University of Bari, Piazza Giulio Cesare 11-70124, Bari, Italy. E-mail: mtrojano@neurol.uniba.it

Potential conflicts of interest: M.T. received honoraria for consultancy or speaking from Sanofi-Aventis, Biogen, and Bayer Schering, and research grants from Merck Serono; F.P. ${ }^{2}$ received honoraria for speaking from Biogen; I.L.S. received honoraria for speaking from Sanofi-Aventis and Biogen, and research grants from Sanofi-Aventis; F.P. ${ }^{3}$ received honoraria for speaking from Sanofi-Aventis and Merck Serono; A.G. received honoraria for speaking from Bayer Schering, Merck Serono, and research grants from Sanofi-Aventis, Biogen Dompè, and Merck Serono; C.P. received honoraria for consultancy or speaking from Sanofi-Aventis, Biogen, Bayer Schering, and Novartis, and research grants from Merck Serono and Sanofi Aventis; A.L. received honoraria for speaking from Biogen and research grants from Sanofi-Aventis, Merck Serono, Biogen, and Bayer Schering; E.M. received honoraria for speaking from Bayer Schering and research grants from Merck Serono, Sanofi-Aventis, Biogen-Dompè, and Bayer Schering; C.A. received honoraria for consultancy or speaking from Sanofi-Aventis, Biogen-Dompè, Bayer Schering, and Merck Serono; M.C. received honoraria for speaking from Bayer Schering; P.L. received research grants from Sanofi-Aventis, Merck Serono, and Biogen; G.C. received honoraria for consultancy or speaking from Teva Neuroscience Plough Corporation and Merck Serono; M.P.A. received honoraria for speaking from Merck Serono and Biogen, and research grants from Sanofi-Aventis, Merck Serono, Biogen, and Bayer Schering. The other authors declare that they have no conflicts of interest.

Received Feb 20, 2009, and in revised form May 11. Accepted for publication May 18, 2009. Published online in Wiley InterScience (www.interscience.wiley.com). DOI: 10.1002/ana.21757 
worsening, which is the hallmark of the late phase of this disease, was also recently shown in a large-scale Italian observational study. ${ }^{4}$ However, whether or not even greater benefits could have been obtained through a treatment initiated earlier in the course of the disease remains an open question.

Histopathological ${ }^{5}$ and magnetic resonance imaging $(\mathrm{MRI})^{6}$ studies suggest that axonal loss occurs during the early inflammatory stages of MS and decreases over time. Natural-history studies have identified a relationship between clinical ${ }^{7-9}$ and MRI $^{10,11}$ features during the early years and long-term disability. Multicenter RCTs ${ }^{12-14}$ that have analyzed early IFN $\beta$ treatment in patients with a clinically isolated syndrome (CIS) suggestive of MS, report significant benefits in delaying further attacks at 2 years, and these benefits were sustained for up to 5 years. ${ }^{15}$ More recently, the openlabel follow-up phase ${ }^{16}$ of the original BENEFIT study ${ }^{14}$ demonstrated a higher effect of early vs. delayed IFN $\beta$ treatment on later accumulation of disability at 3 years. The conclusion from this clinical research is that the earlier treatment is initiated, the better the short-term outcome.

However, at present, a very important unresolved issue for practicing neurologists is to establish if early initiation of treatment with IFN $\beta$ is more efficacious than delayed treatment for preventing the development of long-term confirmed disability, which is ultimately the most important goal of treatment in MS. Another crucial question is how early should treatment be initiated in order to obtain the greatest benefit on longterm outcomes?

This work presents results from a prospective observational study that was conducted to evaluate the impact of early vs. delayed IFN $\beta$ treatment on long-term disability progression in a large cohort of 2,570 RRMS patients prospectively followed for up to 7 years in 15 Italian MS Centers. The main objective was to assess the optimal time to initiate IFN $\beta$ with regard to when the greatest beneficial effect on clinical outcomes was observed.

\section{Patients and Methods}

A cohort of 2,570 IFN $\beta$-treated RRMS was prospectively followed for up to 7 years in 15 Italian MS Centers. The median follow-up time was 4.5 years. Clinical and therapeutic information was recorded according to a computerized and standardized protocol (iMED). A diagnosis of MS was established according to the Poser $^{18}$ and McDonald ${ }^{19}$ online.

The dates of MS onset, first IFN $\beta$ administration, and assignment to irreversible 1-point progression in Expanded Disability Status Scale (EDSS) ${ }^{20}$ score and EDSS 4.0 and 6.0 milestones were systematically assessed for each patient. The EDSS score was recorded at baseline and at least every 6 months thereafter. An EDSS score was defined as irreversible when it persisted for at least 6 months and all the subsequent scores assessed during the follow-up of the patient were ei- ther equal to or higher than that score. During the study period, four preparations of IFN $\beta$ were available: IFN $\beta-1 b$ (Betaferon ${ }^{\circledR} 250 \mu \mathrm{g}$ subcutaneously [SC] every other day) and IFN $\beta$-1a (Avonex ${ }^{\circledR} 30 \mu \mathrm{g}$ intramuscularly [IM] once weekly; Rebif ${ }^{\circledR} 22 \mu \mathrm{g}$ SC three times weekly; Rebif $44 \mu \mathrm{g}$ SC three times weekly). Periods of treatment with IFN $\beta$ were recorded for each patient, including the start and stop dates. The time spent receiving IFN $\beta$ therapy was calculated for each patient excluding transient discontinuations. The duration of time spent receiving transient combination therapy (eg, IFN $\beta$ and mitoxantrone or corticosteroids) was considered to be the same as administration of IFN $\beta$ alone. In this study, we assumed that different IFN $\beta$ products or transient combinations had equivalent impacts on EDSS progression.

\section{Statistical Analysis}

Baseline characteristics for the IFN $\beta$ early treatment and delayed treatment groups were reported as frequency (percentage), mean \pm standard deviation (SD), and median (range), and compared with Pearson's $\chi^{2}$ test and Mann-Whitney U test for categorical and continuous variables, respectively. Time (in years) from IFN $\beta$ treatment initiation and from date of birth to reaching an EDSS score of 4.0 or 6.0, and a 1-point progression in EDSS score were evaluated. For patients who did not reach the specified endpoint, time was censored at the last follow-up visit. Cox proportional hazards regression models adjusted for propensity score (PS) quintiles, ${ }^{21}$ and for PS quintiles and disease duration were used to assess differences between early vs. delayed treatment groups. A set of PS-adjusted Cox models were calculated according to different early and delayed treatment cutoff points in terms of different duration from disease onset at treatment initiation (within 1 year up to within 5 years). Results are expressed as hazard ratios (HRs) and 95\% confidence intervals (CIs).

PS analysis was used to reduce bias in early and delayed treatment comparisons. Because there were three endpoints at issue, three separate PS logistic regression models were first built to predict the probability to be assigned to IFN $\beta$ early treatment. The models included the following covariates at treatment initiation: age, sex, number of relapses in the last year, EDSS score in quintiles, quadratic and cubic covariate terms, and a set of two-term and three-term interactions between the same predictors. Disease duration did not take part into the PS model building process since the definition of early treatment is based on disease duration itself, and adjustment for age at IFN $\beta$ should already account for any potential effect of this variable .

PS logistic models were selected in a step-wise fashion, and model-building stopped when adequate balance of covariates was achieved. ${ }^{22}$ Residual imbalances of covariates in PS quintiles were assessed at each step with a two-way analysis of variance (ANOVA) where each confounder was considered as an outcome and PS quintiles and treatment as factors. Overlapping of PS between treatment and control groups was also checked, and nonoverlapping subjects were excluded from the analyses. Finally, PS quintiles derived from the definitive logistic models were introduced in the Cox models to allow unbiased treatment comparisons. In addition to further eliminate any concerns related to the po- 


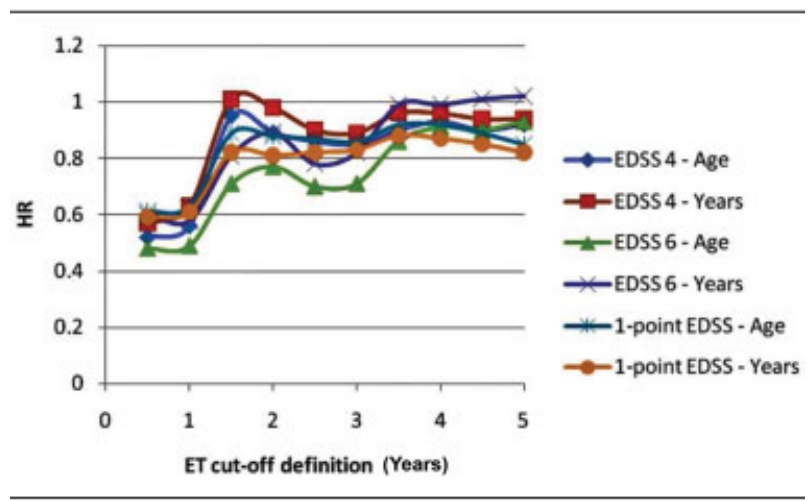

Fig 1. Graphical trends of the propensity score quintiles-adjusted hazard ratios (HRs) according to different early treatment (ET) cutoff points (within 1 year up to within 5 years from disease onset) and to both survival times (years from IFN $\beta$ assignment to endpoint and age at endpoint) for the risk of EDSS 4.0, EDSS 6.0, and 1-point progression in EDSS score. [Color figure can be viewed in the online issue, which is available at www.interscience.wiley.com]

tential role of differential disease duration in early and late treatment groups, PS-adjusted Cox models were also adjusted for disease duration as a linear covariate. The adjustment for duration as a linear covariate was chosen after exploring, on the same endpoints, different forms of adjustment in terms of goodness-of-fit (namely, Akaike's information criterion) in a cohort of untreated patients previously analyzed. ${ }^{4}$

The proportional hazards assumption was checked by graphical inspection of $\log (-\log$ [survival] $)$ plot and assessing the consistency of the HRs in the PS-adjusted Cox models censored to shorter follow-up time frames (from 6 years back to only 3 years of follow-up). This analysis also allowed for the exclusion of the differences in the dropout mechanism between early treatment and delayed treatment patients. Further, since PS methodology only addresses imbalances due to measured confounders, we also performed a sensitivity analysis ${ }^{23}$ on positive findings to account for potential residual confounding due to an unmeasured confounder.

$p$-Values $<0.05$ were considered significant. All the analyses were performed using the Statistical Analysis System (SAS) Package, Release 9.1 (SAS Institute, Cary, NC).

\section{Results}

A plot of the PS quintiles adjusted HRs according to different early treatment cutoff points (within 1 year from disease onset to within 5 years from disease onset) for the risk of reaching EDSS 4.0 and 6.0 milestones and a 1-point progression in EDSS score with both survival times is shown in Fig 1. The lowest HRs for the three PS quintiles-adjusted models were observed with a cutoff of $\leq 1$ year. HRs for times from IFN $\beta$ treatment initiation and from date of birth to reaching an EDSS score of 4.0 were 0.63 and 0.56 ; to reaching an EDSS score of 6.0 were 0.58 and 0.49 ; and to reaching a 1 -point EDSS score progression were 0.61 and 0.63 , respectively. Based on these results, for subsequent analyses, early treatment was defined as $\leq 1$ year from disease onset and delayed treatment was defined as $>1$ year from disease onset.

Fifteen percent $(\mathrm{n}=47)$ and $85 \%(\mathrm{n}=263)$ of patients in the early treatment group $(\mathrm{n}=310)$, and $12 \%(\mathrm{n}=271)$ and $88 \%(\mathrm{n}=1,989)$ of those in the delayed treatment group $(n=2,260)$ had a diagnosis of definite MS according to the McDonald et al. ${ }^{19}$ and Poser et al. ${ }^{18}$ criteria, respectively. Forty percent ( $\mathrm{n}=$ 124), 37\% ( $\mathrm{n}=114), 16 \%(\mathrm{n}=50)$, and $7 \%(\mathrm{n}=$ 22) of 310 patients in the early-treatment group and $36 \%(\mathrm{n}=814), 38 \%(\mathrm{n}=859), 18 \%(\mathrm{n}=407)$, and $8 \%(\mathrm{n}=181)$ of 2,260 patients in the delayedtreatment group received, at their first prescription, Avonex, Rebif $22 \mu \mathrm{g}$, Rebif $44 \mu \mathrm{g}$, and Betaferon, respectively. Only $2.5 \%(\mathrm{n}=8)$ of the 310 patients in the early-treatment group and $3.4 \%(n=77)$ of the 2,260 patients in the delayed-treatment group were, also, treated with mitoxantrone (median length of exposure $=0.6$ years; range $=0.15-1.0)$.

Table 1 presents the baseline characteristics according to treatment group (early-treatment vs. delayedtreatment) for the overall sample. Patients in the earlytreatment group were significantly $(p<0.0001)$ younger $(28.8 \pm 8.3$; mean $\pm S D)$, had a higher number of relapses in the last year $(1.8 \pm 0.9)$ and a lower EDSS score $(1.8 \pm 0.9)$ than those in the delayed group $(27.0 \pm 8.6,1.2 \pm 0.9$, and $2.3 \pm 1.0$, respectively). The percentage of females was greater $(p<$ $0.0026)$ in the early $(76.5 \%)$ than in the delayed $(68.0 \%)$ group. These significant differences justified the use of PS-adjusted comparisons for all the analyses. Due to nonoverlapping propensity score, from $1 \%$ to $3 \%$ of patients were excluded from the analyses. The final sample sizes were 2,277 patients for the EDSS 4.0, 2,570 for the EDSS 6.0, and 2,396 patients for the 1-point EDSS progression outcome.

PS quintiles-adjusted Cox models results are shown in detail for the $\leq 1$ year early treatment cutoff (Table $2)$. Early treatment significantly reduced, by approximately $40 \%$, the risk of reaching the EDSS $4.0(p=$ $0.015, p=0.053)$ and 1-point EDSS progression $(p<0.002)$ compared with delayed treatment; there was a trend $(p=0.09)$ to reduce the risk of reaching EDSS 6.0 with early treatment vs. delayed treatment.

Further adjustment for disease duration of PS quintiles-adjusted Cox models did not materially change our findings. HRs for times from IFN $\beta$ treatment initiation and from date of birth to reaching an EDSS score of 4.0 were 0.49 and 0.70 (95\% CI $=0.30-$ 0.78 and $0.43-1.12 ; p=0.0028$ and 0.13 , respectively); to reaching an EDSS score of 6.0 were 0.43 and 0.63 (95\% CI $=0.19-1.01$ and $0.27-1.47 ; p=0.052$ and 0.28 , respectively); and to reaching a 1 -point progression in EDSS score were 0.52 and 0.66 (95\% 


\begin{tabular}{|c|c|c|c|c|}
\hline Variable & Early $(\mathrm{n}=310)$ & Delayed $(n=2,260)$ & All $(n=2,570)$ & $p$-Value \\
\hline \multicolumn{5}{|c|}{ Age at treatment initiation (years) } \\
\hline Mean $\pm S D$ & $28.8 \pm 8.3$ & $34.2 \pm 9.1$ & $33.5 \pm 9.2$ & \\
\hline Median (range) & $27.4(6.3-59.2)$ & $33.4(4.4-61.4)$ & $32.8(4.4-61.4)$ & $<0.0001$ \\
\hline \multicolumn{5}{|l|}{ Age at onset (years) } \\
\hline Mean \pm SD & $28.2 \pm 8.3$ & $27.0 \pm 8.6$ & $27.1 \pm 8.6$ & \\
\hline Median (range) & $26.8(5.9-58.9)$ & $25.7(1.4-57.7)$ & $26.0(1.4-58.9)$ & 0.0094 \\
\hline \multicolumn{5}{|c|}{ Disease duration (years) } \\
\hline Mean \pm SD & $0.6 \pm 0.3$ & $7.1 \pm 5.8$ & $6.3 \pm 5.8$ & \\
\hline Median (range) & $0.6(0.1-1.0)$ & $5.4(1.0-42.2)$ & $4.6(0.0-42.2)$ & 0.0001 \\
\hline \multicolumn{5}{|l|}{ EDSS score } \\
\hline Mean \pm SD & $1.8 \pm 0.9$ & $2.3 \pm 1.0$ & $2.16 \pm 1.0$ & \\
\hline Median (range) & $1.5(1.0-5.5)$ & $2.0(1.0-5.5)$ & $2.0(1.0-5.5)$ & $<0.0001$ \\
\hline \multicolumn{5}{|c|}{ Number of bouts in the last year } \\
\hline Mean \pm SD & $1.8 \pm 0.9$ & $1.2 \pm 0.9$ & $1.3 \pm 0.9$ & \\
\hline Median (range) & $2.0(0.0-6.0)$ & $1.0(0.0-6.0)$ & $1.0(0.0-6.0)$ & $<0.0001$ \\
\hline Sex, female, n (\%) & $237(76.5)$ & $1537(68.0)$ & $1774(69.0)$ & 0.0026 \\
\hline \multicolumn{5}{|c|}{$\begin{array}{l}\text { *Data are reported as number (percentage), mean } \pm S D \text {, and median (range); } p \text {-values refer to Pearson's } \chi^{2} \text { test and Mann-Whitney } U \\
\text { test for categorical and continuous variables, respectively. } \\
\text { MS = multiple sclerosis; early }=\leq 1 \text { year from disease onset; delayed }=>1 \text { year from disease onset; } S D=\text { standard deviation; } \\
\text { EDSS = Expanded Disability Status Scale. }\end{array}$} \\
\hline
\end{tabular}

$\mathrm{CI}=0.39-0.70$ and $0.49-0.89 ; p=0.0001$ and 0.0068 , respectively).

PS-adjusted estimated survival curves, which graphically translated risk reductions expressed by HRs, showed that early treatment slowed the time to reach an EDSS of 4.0 and a 1-point progression in EDSS progression (Fig 2). The estimated percentage of patients that would reach EDSS 4.0 after a median follow-up of 4.5 years was $33.5 \%$ for the delayed treatment group vs. $23.2 \%$ for early treatment. The $23.2 \%$ threshold was reached with a delay of 19.5 months $(84$ months for the early-treatment group and 64.5 months for the delayed-treatment group). About $60 \%$ of delayed treatment patients compared with $42.6 \%$ of the early treated group would reach a 1-point EDSS progression after 4.5 years. The $42.6 \%$ threshold was reached with a delay of 14 months (84 months for the early-treatment group and 70 months for the delayedtreatment group).

A sensitivity analysis (Table 3) was performed for the two significant endpoints to assess the robustness of our findings. For the risk of reaching an EDSS milestone of 4.0, the significant effect of early treatment might be altered by an unmeasured confounder with

Table 2. PS Quintiles Adjusted Cox Models for Time from IFN及 Assignment and from Date of Birth (Age at Endpoint) To Reach the Three Endpoints by Early Treatment Within 1 Year of Disease Onset

\begin{tabular}{|c|c|c|c|c|}
\hline \multirow[t]{2}{*}{ Endpoint } & \multirow[t]{2}{*}{ Survival Time (years) } & \multicolumn{3}{|c|}{ PS Quintiles } \\
\hline & & $\mathbf{H R}^{\mathrm{a}}$ & 95\% HR CI & $p$ \\
\hline \multirow[t]{2}{*}{ EDSS 4} & Age at endpoint & 0.56 & $0.36-0.90$ & 0.0150 \\
\hline & Years from IFN $\beta$ to endpoint & 0.63 & $0.40-1.01$ & 0.0529 \\
\hline \multirow[t]{2}{*}{ EDSS 6} & Age at endpoint & 0.49 & $0.22-1.13$ & 0.0936 \\
\hline & Years from IFN $\beta$ to endpoint & 0.58 & $0.25-1.33$ & 0.1979 \\
\hline \multirow[t]{2}{*}{ EDSS 1-point progression } & Age at endpoint & 0.63 & $0.48-0.85$ & 0.0019 \\
\hline & Years from IFN $\beta$ to endpoint & 0.61 & $0.46-0.82$ & 0.0009 \\
\hline
\end{tabular}

${ }^{\text {a}} \mathrm{HR}<1$ favors early treatment.

$\mathrm{PS}=$ propensity score; $\mathrm{HR}=$ hazard ratio; IFN $=$ interferon; EDSS = Expanded Disability Status Scale; CI $=$ confidence interval. 

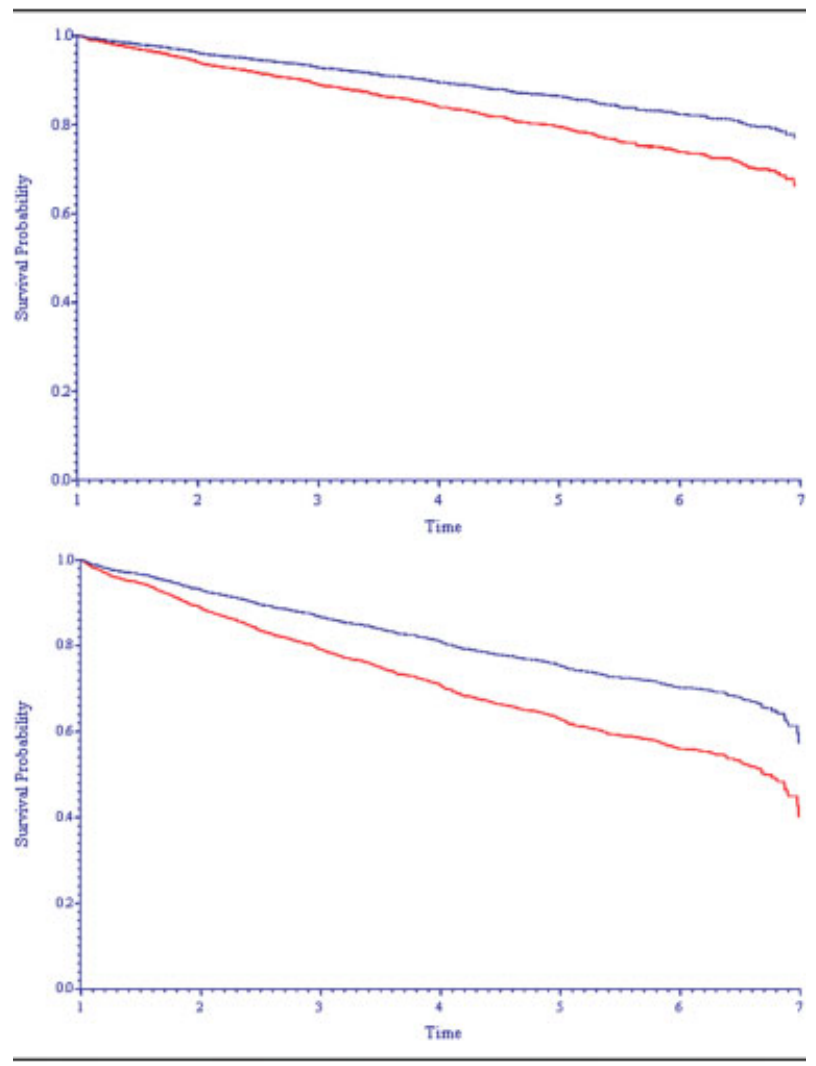

Fig 2. Propensity score-adjusted survival curves for time from treatment initiation to reach (A) confirmed EDSS 4.0 score, and $(B)$ a 1-point progression in EDSS score. Survival probability represents the estimated proportion of patients who did not reach the endpoint. Continuous line $=$ delayed treatment group; dotted line = early treatment group. [Color figure can be viewed in the online issue, which is available at www. interscience.wiley.com.]

an $\mathrm{HR}=1.5$ and a prevalence imbalance between the early-treatment group and the delayed-treatment group $\left(\mathrm{P}_{0}-\mathrm{P}_{1}\right)$ of at least $30 \%$ or with a prevalence imbalance of $20 \%$ but an $\mathrm{HR}=2.0$ or with a prevalence imbalance of at least $10 \%$ but an $\mathrm{HR}=2.5$. For the risk of a 1-point progression in EDSS score, an unmeasured confounder with an HR $=1.5$ and a $50 \%$ prevalence imbalance or with a prevalence imbalance of at least $30 \%$ but an $\mathrm{HR}=2.0$ or with a prevalence imbalance of at least $20 \%$ but an HR $=2.5$ would be sufficient to alter the significant effect of early treatment.

\section{Discussion and Conclusions}

In this exploratory analysis we assessed whether longerterm disability of RRMS patients would benefit from earlier initiation of treatment with IFN $\beta$. The availability of a large sample of patients, followed for up to 7 years by 15 experienced Italian MS centers, allowed us to prospectively evaluate the impact of early vs. delayed IFN $\beta$ treatment on the natural course of MS.
The results show that patients treated early respond better than those who initiated the treatment later. Early IFN $\beta$ treatment was associated with a significant reduction in the risk of reaching an EDSS milestone of 4.0 and the risk of a 1-point progression in EDSS score, and with a trend suggestive of reduction in risk of reaching an EDSS milestone of 6.0 (likely not statistically significant due to a low number of events during the follow-up) when compared with delayed treatment. More interestingly, we found that the greatest difference in treatment benefit was observed between patients who received IFN $\beta$ within the first year from disease onset in comparison with those who received the treatment after this time.

Our data followed by a median of 4.5 years are consistent with the 3-year results of the open-label follow-up phase ${ }^{16}$ of the original BENEFIT study, ${ }^{14}$ which showed a slower disease progression in patients treated continuously with IFN $\beta-1 \mathrm{~b}$ vs. those treated with placebo for the first 1 or 2 years, and with the 4-year results of the PRISMS study ${ }^{24}$ showing time to EDSS progression was longer in those patients who had been initially randomized to IFN $\beta-1$ a than in those in the crossover group who had received placebo during years $1-2$. It is noteworthy that in the former study ${ }^{16}$ the early treatment at 3 years reduced the risk for the progression of 1 point in the EDSS score, confirmed at 6 months, compared with delayed treatment, by the same rate $(40 \%)$ of reduction that we found at 4.5 years. Moreover, in the latter study ${ }^{24}$ the delay (11-18 months) to first confirmed EDSS progression observed in the earlier IFN $\beta-1 \mathrm{a}-22 \mu \mathrm{g} / 44 \mu \mathrm{g}$-treated

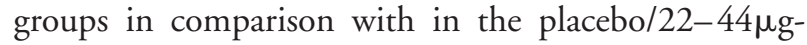
treated crossover groups was similar to that we found (14 months) between early- and delayed-treatment patients. In addition, in our study, the extremely larger sample size ( $\mathrm{n}=2,570$ RRMS patients) and the longer follow-up in comparison with those in the BENEFIT ${ }^{16}$ and PRISM- $4^{24}$ trials, and the adjustment for selection bias, by using propensity score, add considerable value to this particular analysis. Although RCT is undoubtedly the ideal way for providing evidence on drug efficacy, results derived from open-label extensions of RCTs are not necessarily better than those obtained by long-term observational studies with rigorous study design or statistical analysis. ${ }^{25}$ Indeed, the methodological rigor (randomization) of the initial RCTs is often eroded during the extension phase ${ }^{26}$ because the number of patients often decreases dramatically and the data might be collected unblinded. Moreover, observational clinical data have the advantage to be more representative of the MS population than RCT study samples. Finally, the most important finding is that we were able to perform a cutoff analysis to assess the optimal time to initiate IFN $\beta$ treatment with regard to the greatest benefits on long-term disability progres- 


\begin{tabular}{|c|c|c|c|c|}
\hline Endpoint & $\mathbf{H R}^{\mathbf{a}}$ & $P_{1}-P_{0}^{b}$ & HR & $95 \% \mathrm{CI}$ \\
\hline \multirow[t]{3}{*}{ EDSS 4.0} & 1.5 & 0.3 & 0.64 & $0.41-1.03$ \\
\hline & 2 & 0.2 & 0.66 & $0.43-1.06$ \\
\hline & 2.5 & 0.1 & 0.63 & $0.41-1.02$ \\
\hline \multirow[t]{3}{*}{ EDSS 1-point progression } & 1.5 & 0.5 & 0.76 & $0.57-1.02$ \\
\hline & 2 & 0.3 & 0.78 & $0.59-1.04$ \\
\hline & 2.5 & 0.2 & 0.77 & $0.58-1.03$ \\
\hline \multicolumn{5}{|c|}{$\begin{array}{l}\text { *This analysis assumes that (1) the unmeasured confounder is binary, (2) the unmeasured confounder is independent of measured } \\
\text { confounders, and (3) no interaction occurs between the unmeasured confounder and exposure. } \\
\text { a Hypothetical HR of the unmeasured confounder on time to endpoints. } \\
\text { bDifferences in prevalence of the unmeasured confounder between early vs. delayed treatment groups. } \\
\text { HR = hazard ratio; EDSS = Expanded Disability Status Scale; CI = confidence interval. }\end{array}$} \\
\hline
\end{tabular}

sion. This kind of analysis is easily obtained with an observational design, but would be infeasible and unethical within a clinical trial setting. That is, no patients could be forced to any treatment arm with a prespecified delay time.

The delay in accumulation of disability with early treatment, seen in this study, is in line with neuropathological, clinical, and MRI findings ${ }^{5-11,27,28}$ showing that processes which lead to irreversible disability actually begin very early in the course of the disease. Such damage, primary or secondary to inflammation, may be irreversible, and there is strong evidence to suggest that efficacy lost as a consequence of delay in the onset of treatment cannot be regained. ${ }^{29}$ Moreover, the extent of the benefits that can be obtained from a treatment with IFN $\beta$ decreases in patients with secondary progressive MS. ${ }^{30,31}$ Furthermore, natural history studies demonstrated that the early course of disease can influence long-term outcome. The frequency of relapse and the interval between relapses during the first 2 years, ${ }^{7}$ incomplete recovery from relapses during the first 5 years, ${ }^{9}$ and the degree of disability after 5 years have been associated with the development of disability up to 25 years later. ${ }^{8}$ Moreover, the number and volume changes in MRI lesions seen in the first 2 to 5 years of disease course correlate with the degree of disability in the longer-term. ${ }^{10,11}$ It therefore seems logical that effective treatment should be initiated early in the disease course to early inhibit the cascade of events that leads to irreversible axonal damage and disability. Our results strongly support this hypothesis.

In this study, we estimated the effect of IFN $\beta$ also by age at endpoints because recent reports suggested that the accumulation of irreversible disability in MS appear to be, at least in part, an age-dependent process $^{32}$ and survival techniques accounting for the assessment of ages at reaching irreversible disability end points may provide more accurate target outcomes for therapeutic trials. ${ }^{33}$
However, the limitations of this observational study merit discussion. As with all observational studies, the major issue of was the lack of randomization and therefore potential selection bias. ${ }^{34-36}$ The treatment groups were imbalanced for all of the baseline covariates. Particularly, the early-treatment group included MS patients with a more active course and a faster disability progression (median number of relapses $=2$ and median EDSS score $=1.5$ in a median disease duration of less than 1 year) than the delayed group (median number of relapses in the last year $=1$ and median EDSS score $=2$ in a median disease duration of 5.4 years). This meant that it was necessary to use statistical methods to adjust the comparisons. Therefore, we used the most common approach to overcome this issue in treatment comparisons in observational studies; ie, a PS-adjusted analysis. ${ }^{21,22}$ This technique has already been used to test drug effects in other therapeutic areas $^{37,38}$ and also in MS. ${ }^{4}$ PS analysis, taking into consideration parameters of interest (age, sex, EDSS score, and number of relapses in the last year prior to the start of treatment) that would likely affect the outcome, allowed us to obtain two balanced groups of patients who have similar likelihoods of receiving an early therapy, and resembling randomized cohorts of patients. Moreover, although we checked externally on the untreated cohort of a previously analyzed dataset ${ }^{4}$ that age at IFN $\beta$ already takes into account any potential effect of disease duration (ie, for duration defined as $\leq 1$ year vs. $>1$ year, the HRs for time to reaching EDSS 4 were 0.76 [unadjusted], 1.23 [age-adjusted], and 1.82 [duration-adjusted]) a clearer assessment of early treatment unconfounded by disease duration was also reported, showing that the consistency of results was retained.

An important limitation of the PS approach is that it cannot adjust for variables that are not measured in a study (such as MRI variables in this study); therefore, we conducted a sensitivity analysis ${ }^{23}$ to evaluate their 
possible impact on the study outcome. The results of this analysis showed that the positive effect of early IFN $\beta$ treatment for the endpoints 1-point EDSS progression and risk of reaching an EDSS of 4.0 appeared sensitive to small bias, but their HRs were still suggestive of a slower disease progression in comparison with a delayed treatment. In our study, hidden bias might reflect the inability to account for factors related to physicians. Absence of blinding, for instance, might affect EDSS assessment at each visit. However, even though findings that could be sensitive to small unmeasured confounders should be interpreted with caution in a disproof approach, sensitivity to small biases is not a sufficient reason to dismiss such findings.

Moreover, once overt and hidden bias are taken into account, any attempt to assess treatment effectiveness in "real-world" settings within the framework of properly conducted observational studies should not be dismissed a priori. An enhanced quality of observational studies may provide the opportunity for a less expensive evaluation of therapies in clinical medicine.

The key findings from this study, already demonstrated in clinical trials with shorter follow-up, ${ }^{16,24}$ are that patients who begin treatment later do not reap the same long-term benefits as those who begin treatment earlier during the disease course and that the first year from disease onset seems to represent the time frame when we could expect that initiation of an effective treatment would allow subsequent accumulation of disability to be minimized.

Since patients had some clinical heterogeneity, it could be useful for clinical practice to further analyze the long-term benefit of early-stage IFN $\beta$ treatment in different patient subgroups.

\section{Italian Multiple Sclerosis Database Network (MSDN) Group}

List of Other Collaborators: E. Di Monte, P. Iaffaldano, V. Direnzo, M. D'Onghia (Department of Neurology, University of Bari, Bari, Italy); S. Lo Fermo, S. Messina, E. D'Amico (Department of Neurology, University of Catania, Catania, Italy); A. Rizzo, M. Zaffaroni (MS Centre, Hospital of Gallarate, Gallarate, Italy); E. Portaccio (Department of Neurology, University of Florence, Florence, Italy); V. Martinelli (Department of Neurology, Hospital of San Raffaele, Milan, Italy); L. Prosperini, E. Onesti (Multiple Sclerosis Center, S. Andrea Hospital, University of Rome "La Sapienza," Rome, Italy); P. Aridon , M. D’Amelio, P. Ragonese (Department of Clinical Neurosciences, University of Palermo, Palermo, Italy); G. De Luca, D. Farina, V. Di Tommaso (Department of Oncology and Neuroscience, University G. d'Annunzio, Chieti, Italy); E. Tavazzi, V. Bargiggia, P. Crivelli (Multiple Sclerosis Center, Neurological Institute C. Mondino, Pavia, Italy); A. Cortese, S. Di Rezze, V. Durastanti (Department of Neurological
Sciences, University La Sapienza, Rome, Italy); G. Alfieri (Department of Neurological Sciences, 2nd University of Naples, Naples Italy); I. Rosellini (O.U. Neurology, Ca' Foncello Hospital, Treviso, Italy); and M. Modesto, M. Notariello, P. Tortorella (Department of Medical and Occupational Sciences, University of Foggia, Foggia, Italy).

This work was supported by annual research grants from the Italian University and Research Ministry (MIUR) (COFIN 2005-2006 M.T. Proselto Stzetegieo Regiolle Puque "Nurobío tech" PS 124, M.T., V.L.).

We thank all patients who participated in this study and the Fondazione Internazionale Cesare Serono for providing the iMED software to the Italian MS centers.

\section{References}

1. The IFNB Multiple Sclerosis Study Group. Interferon beta-1b is effective in relapsing-remitting multiple sclerosis. I. Clinical results of a multicenter, randomized, double-blind, placebo controlled trial. Neurology 1993;43:655-661.

2. Jacobs LD, Cookfair DL, Rudick RA, et al. Intramuscular interferon beta-1a for disease progression in relapsing multiple sclerosis. Ann Neurol 1996;39:285-294.

3. PRISMS (Prevention of Relapses and Disability by Interferon b-1a Subcutaneously in Multiple Sclerosis) Study Group. Randomised double-blind placebo-controlled study of interferonb-1a in relapsing/remitting multiple sclerosis. Lancet 1998;352: $1498-1504$.

4. Trojano M, Pellegrini F, Fuiani A, et al. New natural history of interferon- $\beta$-treated relapsing multiple sclerosis. Ann Neurol 2007;61:300-306.

5. Trapp BD, Peterson J, Ransohoff RM, et al.. Axonal transection in the lesions of multiple sclerosis. N Engl J Med 1998; 338:278-285.

6. Kuhlmann T, Lingfeld G, Bitsch A, et al. Acute axonal damage in multiple sclerosis is most extensive in early disease stages and decreases over time. Brain 2002;125:2202-2212.

7. Weinshenker BG, Bass B, Rice GP, et al. The natural history of multiple sclerosis: a geographically based study. 2. Predictive value of the early clinical course. Brain 1989;112(Pt 6): $1419-1428$.

8. Runmarker B, Andersen O. Prognostic factors in a multiple sclerosis incidence cohort with twenty-five years of follow-up. Brain 1993;116:117-134.

9. Kurtzke JF, Beebe GW, Nagler B, et al. Studies on the natural history of multiple sclerosis. 8. Early prognostic features of the later course of the illness. J Chronic Dis 1977;30:819-830.

10. O'Riordan JI, Thompson AJ, Kingsley DP, et al. The prognostic value of brain MRI in clinically isolated syndromes of the CNS. A 10-year follow-up. Brain 1998;121(Pt 3):495-503.

11. Tintoré M, Rovira A, Rio J, et al. Baseline MRI predicts future attacks and disability in clinically isolated syndromes. Neurology 2006;67:968-972.

12. Jacobs LD, Beck RW, Simon JH, et al. Intramuscular interferon beta-1a therapy initiated during a first demyelinating event in multiple sclerosis. CHAMPS Study Group. N Engl J Med 2000;343:898-904.

13. Comi G, Filippi M, Barkhof F, et al. Effect of early interferon treatment on conversion to definite multiple sclerosis: a randomised study. Lancet 2001;357:1576-1582. 
14. Kappos L, Polman CH, Freedman MS, et al. Treatment with interferon beta- $1 \mathrm{~b}$ delays conversion to clinically definite and McDonald MS in patients with clinically isolated syndromes. Neurology 2006;67:1242-1249.

15. Kinkel RP, Kollman C, O'Connor P, et al. IM interferon beta-1a delays definite multiple sclerosis 5 years after a first demyelinating event. Neurology 2006;66:678-684.

16. Kappos L, Freedman MS, Polman CH, et al.; for the BENEFIT Study Group. Effect of early versus delayed interferon beta- $1 b$ treatment on disability after a first clinical event suggestive of multiple sclerosis: a 3-year follow-up analysis of the BENEFIT study. Lancet 2007;370:389-397.

17. Mechati S, Peyro-St-Paul H. iMed: a new electronic database for monitoring patients with multiple sclerosis. Mult Scler 2001;7:S31

18. Poser CM, Paty DW, Scheinberg L, et al. New diagnostic criteria for multiple sclerosis: guidelines for research protocols. Ann Neurol 1983;13:227-231.

19. McDonald WI, Compston A, Edan G, et al. Recommended diagnostic criteria for multiple sclerosis: guidelines from the International Panel on the Diagnosis of Multiple Sclerosis. Ann Neurol 2001;50:121-127.

20. Kurtzke JF. Rating neurological impairment in multiple sclerosis: an expanded disability status scale (EDSS). Neurology 1983;33:1444-1452.

21. D'Agostino RB, Jr. Propensity score methods for bias reduction in the comparison of a treatment to a non-randomized control group. Stat Med 1998;17:2265-2281.

22. Yanovitzky I, Zanutto E, Hornik R. Estimating causal effects of public health education campaigns using propensity score methodology. Eval Prog Plan 2005;28:209-220.

23. Lin DY, Psaty BM, Krommal RA. Assessing the sensitivity of regression results to unmeasured confounders in observational studies. Biometrics 1998;54:948-963.

24. The PRISMS (Prevention of Relapses and Disability by Interferon-b-1a Subcutaneously in Multiple Sclerosis) Study Group, and the University of British Columbia MS/MRI Analysis Group. PRISMS-4: long-term efficacy of interferonb-1a in relapsing MS. Neurology 2001;56:1628-1636.

25. D’Agostino RB, Jr., D’Agostino RB, Sr. Estimating treatment effects using observational data. JAMA 2007;297:314-316.
26. Noseworthy JH. How much can we learn from long-term extension trials in multiple sclerosis? Neurology 2006;67: 930-931.

27. De Stefano N, Narayanan S, Francis GS, et al. Evidence of axonal damage in the early stages of multiple sclerosis and its relevance to disability. Arch Neurol 2001;58:65-70.

28. De Stefano N, Matthews PM, Filippi M, et al. Evidence of early cortical atrophy in MS: relevance to white matter changes and disability. Neurology 2003;60:1157-1162.

29. Schwid S, Bever CT, Jr. The cost of delaying treatment in multiple sclerosis: what is lost is not regained. Neurology 2001;56: 1620 .

30. European Study Group on Interferon Beta-1b in Secondary Progressive MS. Placebo-controlled multicentre randomised trial of interferon beta- $1 \mathrm{~b}$ in treatment of secondary progressive multiple sclerosis. Lancet 1998;352:1491-1497.

31. Secondary Progressive Efficacy Clinical Trial of Recombinant Interferon-Beta-1a in MS (SPECTRIMS) Study Group. Randomized controlled trial of interferon-beta- 1 a in secondary progressive MS: clinical results. Neurology 2001;56:1496-1504.

32. Trojano M, Liguori M, Zimatore GB, et al. Age-related disability in multiple sclerosis. Ann Neurol 2002;51:475-480.

33. Confavreux C, Vukusic S. Age at disability milestones in multiple sclerosis. Brain 2006;129:595-605.

34. Rubin DB. The design versus the analysis of observational studies for causal effects: parallels with the design of randomized studies. Stat Med 2007;26:20-36.

35. Normand SLT, Sykora K, Li P, et al. Readers guide to critical appraisal of cohort studies: 3. Analytical strategies to reduce confounding. BMJ 2005;330:1021-1023.

36. Trojano M. Is it time to recognize the use of observational data to estimate treatment effectiveness in multiple sclerosis? Neurology 2007;69:1478-1479.

37. Kern LM, Powe NR, Levine MA, et al. Association between screening for osteoporosis and the incidence of hip fracture. Ann Intern Med 2005;142:173-181.

38. Austin PC. Propensity-score matching in the cardiovascular surgery literature from 2004 to 2006: a systematic review and suggestions for improvement. J Thorac Cardiovasc Surg 2007;134: $1128-1135$. 